\title{
A new statistical mechanical formalism for gases
}

\author{
René D. Rohrmann* \\ Instituto de Astronomía, UNAM, A.P. 70-264, 04510 México D.F., México
}

(Dated: January 25, 2019)

\begin{abstract}
An equilibrium theory of classical fluids based on the space distribution among the particles is derived in the framework of the energy minimization method. This study is motivated by current difficulties of evaluation of optical properties in atmospheres of degenerate stars. Present paper focuses on diluted one-component systems, where the interaction energy is calculated as a sum of binary contributions. The spatial configuration of the gas is described in terms of a particle-state variable $v$ which roughly measures the free space surrounding each particle. The formalism offers a unified treatment of both thermodynamics and structure of fluids, since it not only provides the state function (the Helmholtz free energy) of a fluid, but also automatically gives information on the microstructure of the system (e.g. the nearest-neighbor distribution function). Equations of state and nearest-neighbor distribution functions of perfect and hard-body fluids are obtained in straightforward way in one, two and three dimensions. The formalism is applied to describe the atomic population in a partially ionized hydrogen gas. Combined and self-consistent evaluations of atomic populations and internal effects on bound states are performed in a detailed form. The present theory allow us to resolve the atomic density at each internal level into groups of atoms which experiment different perturbation intensities according to the size of their spaces $v$.
\end{abstract}

PACS numbers: 02.50.-r, 05.20.Jj, 51.30.+i

\section{INTRODUCTION}

The evaluation of the thermodynamical, transport and optical properties of non-ideal plasmas is of great interest to characterize the behavior of the matter at different physical conditions. It has many applications in material sciences, geophysics and astrophysics. In particular, the equations of state provide the basic thermodynamic quantities needed to determine the physical properties of stellar envelopes and interiors. Models of gases must also provide detailed atomic and molecular populations required to obtain the monochromatic opacity, a quantity which is essential to calculate realistic spectra emitted from astrophysical objects. Our study is motivated by current difficulties of evaluation of gas opacities in envelopes of white dwarf stars (WDs).

While non-ideal effects on equations of state (e.g., departures from the ideal $P-T-\rho$ relationship) become present at densities near to ionization pressure (which occurs above $\rho \approx 0.3 \mathrm{~g} \mathrm{~cm}^{-3}$ for a pure hydrogen plasma 1]), signatures of non-ideal effects (i.e., particle interactions) are evident in spectra emitted by gases at very low densities. As it is well-known since the earliest days of the quantum theory, interparticle perturbations are responsable for the reduction of the ionization energies, shifts of lines and modifications of their profiles [2].

The reduction of the ionization potential along with the line merging, yields the advance of the series limits in the spectrum and forms 'pseudocontinuums', which resemble extensions of the bound-free adsorptions toward longer wavelength [3]. Pseudocontinuum opacities may

*Present address: Observatorio Astronómico, UNC, Laprida 854, (5000) Córdoba, Argentina produce strong modifications in the spectra of cool WDs with rich-hydrogen envelopes [4, 5]. However, so far, the model atmospheres fail to reproduce in detail the photometric observations of WDs with effective temperatures below $4000 \mathrm{~K}$, and the source of discrepancies likely lies in the computation of gas opacities which are affected by non-ideal effects [6].

Current optical simulations [7, 8], commonly used in the evaluation of WD spectra (e.g. [4, 5, 9, 10]), are based on the occupation probability formalism elaborated by Hummer and Mihalas (HM) 11]. On the basis of earlier works [12] and the implementation of the technique of free energy minimization, the HM formalism assigns to each atomic state $j$ a probability $w_{j}$ of finding the atom in this state relative to that of finding it in a similar ensemble of non-iteracting particles. For the computation of optical properties, the quantity $w_{j}$ is considered as an estimate of the number of states of type $j$ that are available to be occupied (bound level), whereas $1-w_{j}$ is a measure of the fraction of $j$-states that are severely perturbed by plasma interactions, such that an atom in this fraction is actually unbounded (dissolved level) 7]. In this heuristic scheme, a radiative excitation of an atom from a bound level to a dissolved level constitutes a pseudo-continuum absorption. Althought this proposal seems intuitively right, we have recently showed that it is not self-consistent with the HM formalism [10]. Briefly, the optical simulations assume the existence of a fraction of atoms with perturbed electronic energies (values shifted relative to the continuum), whereas the occupation HM formalism has been developed for particles species which have unperturbed energies (ionization potential of isolated atom). As a consequence, the atomic population which contributes to a pseudocontinuum absorption is overvaluated, because the abundances of perturbed atoms are calculated with lower bound ener- 
gies than those assumed in their transitions to dissolved states. This may explain why such optical simulations yield a huge (unphysical) pseudocontinuum-Lyman opacity for cool WDs [5]. Since the starting level of these processes is the fundamental state, the overevaluation of the Lyman pseudocontinuum is more severe than those of Balmer and Paschen.

While a group of gas properties (equations of state and response functions, e.g., specific heats) may be calculated directly from the global distribution of particles (mean densities), others, such as emissivities and opacities in general depend on particular population subsets. As an appropriate example, we mention the point of view adopted by the statistical theory of line broadening by pressure effects. There, the spectrum of a broadened line is determinated from a statistical sum, where the contribution of each radiating atom depends on the configuration of its surrounding particles [13]. This picture can be also applied to continuum transitions. Thus, an appropriate treatment of pseudo-continnum opacities could be developed on the basis of a formalism able to distinguish groups of atoms which, at a given instant, experiment different degrees of interparticle perturbation.

Motivated by these considerations, we believe it is physically significant and useful to develop a statistical mechanical formalism for gases based on the distribution of the physical space among the particles. The central idea is that to each particle is assigned a space region $v$ nearly related with the closeness of particle neighbors, and which is treated as a configurational parameter in the thermostatistical description of the gas. A particle with small space $v$ will have very near neighbors and, therefore, it will be subjected on the average to high perturbations, while a particle will be roughly isolated if it has a huge space $v$ assigned. This description offers an appropriate framework for the study of optical properties of gases. So far, the application of statistical mechanics in the analysis of space partition into a set of volumes has been circumscribed to some particular systems such as cristals 14. Similar theory for gases has not been formulated as far as we are aware. As a first step, the formalism developed in this paper is devoted to one-component gases at the limit of low densities.

We formulate the theory in terms of the widely used free-energy minimization method [15]. Our aim is therefore to develop a Helmholtz free-energy model for gases which contains a notion of space per particle, and to find the statistical equilibrium state and the thermodynamic properties of the system from the energy minimization with respect to the particle number density, subjected to specific conditions. Since the present work concerns gases at low densities, we assume factorization of the free energy into configurational, translational and particleinteraction contributions.

The paper is organized as follows. Sec. П briefly reviews the importance of spatial statistical in numerous research areas, and focuses on the application of space partition schemes to the analysis of spatial micro-structures in gases. In Sec. IIII we introduce the volume distribution among particles as a new configurational parameter in thermostatistics of gases. The gas is treated with the Maxwell-Boltzmann statistics and the particle interactions are represented by additive pair potentials. The equilibrium states are derived in Sec. [IV In Secs. VVIVI the theory is applied to perfect and hard-sphere gases. We show that they have the expected thermodynamic properties. There, the Van der Waals model is introduced as an illustration of some of the considerations underlying this article. In Sec. VII we explore the application of the theory to the description of atoms perturbed by plasma effects in a partially ionized, hydrogen gas. Concluding remarks are given in Sec. VIII

\section{SPATIAL STATISTICS}

One of the most basic properties of a collection of objects is the division of space among them. The partition of space is commonly associated to processes that create random geometries. There is a considerable variety of physical systems which show stochastic structures. For example: biological tissues 16]; landscape geometries in agriculture and forestry [17]; territoriality zoology [18]; grains in foams and metallurgial aggregates [14, 19]; intermolecular cavities in fluids [20]; solar granulation [21]; fragmentation of interestellar clouds 22]; stellar populations [23]; matter and void distributions in the large-scale structure of the Universe 24]. Most of these systems have often been studied with computer simulation of stochastic, space-filling structures. A variety of geometric constructions have been applied in these analyses [25].

Gases in thermodynamic equilibrium are also typical disordered systems. In fact, althougth macroscopically homogeneous in the absence of external fields, these systems have spatial inhomogeneities at microscopic levels. The particles of a fluid move continuously and the instantaneous spatial concentration of material is non-uniform and fluctuating at different scales. Fig. [1 (above on the left) shows a group of objects random distributed in a surface, which represents a typical configuration of a twodimensional ideal gas at a certain instant. We note that some particles are close enough among them while others are separated from their nearest neighbors by larger distances. One can then imagine a division of the space into a set of regions, one region per particle taking into account the closeness of its neighbors. The most likely well-known partition of space is given by the Voronoï tesselation [26]. The Voronoi region of a given object is the collection of points in space nearer to that object than to any other. The complete set of Voronoï regions form the so-called Voronoï diagram of the system. An example is given in Fig. 11 (above on the right). There are, however, many other types of space divisions (e.g., generalizations of the Voronoï tesselations [27]).

It should be also observed that while the particle distribution of a perfect gas corresponds to a Poisson dis- 

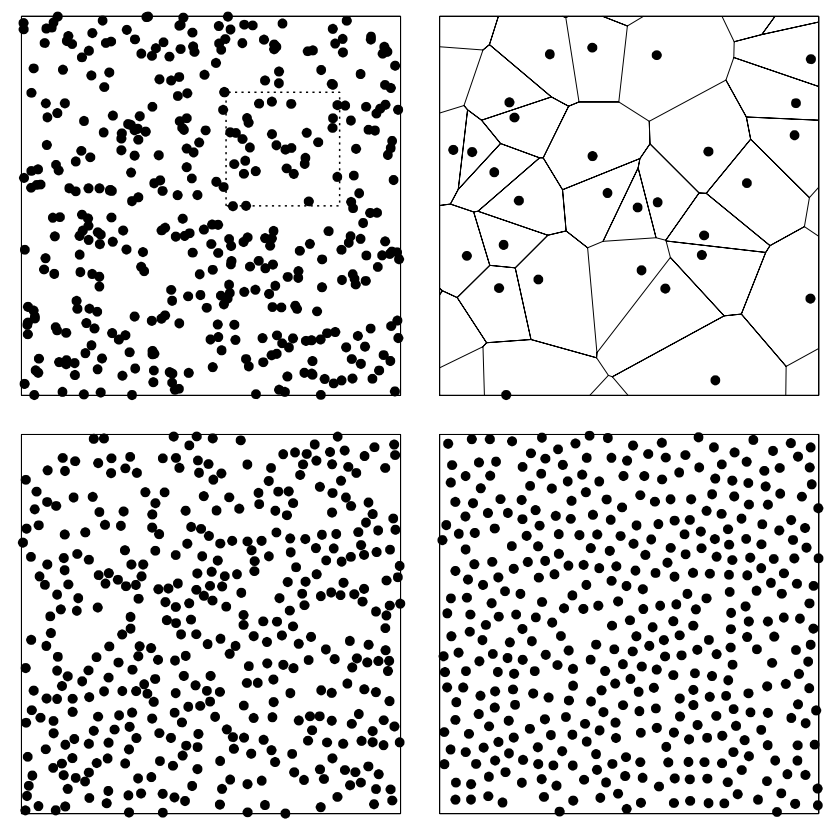

FIG. 1: Above: Poisson distribution of 400 points in a plane (on the left) and the Voronoi tessellation corresponding to points within the dotted box (on the right). Solid lines denote the boundaries of the region of space closest to each particle. Below: Typical configuration of centers of 400 hard discs for a packing fraction (fraction of surface covered by the discs) $\eta=0.2$ (on the left) and $\eta=0.5$ (on the right).

tribution of points in the space, gases formed by interacting particles are characterized by other different distributions. Thus, for example, the well-known one component plasma (model of discrete positive charges in a continuous negatively charged background [28]) shows a long-range ordered (or 'superhomogenous') arrangement of particles, which can be appreciated from the analysis of the so-called power spectrum of the particle distribution [29]. Also the hard-body fluids show particular spatial patterns which have been studied extensively 30]. As an example, the lower panels in Fig. 1 show typical configurations of hard-disc fluids. It is evident that the density fluctuations of a gas in equilibrium depend on the type of interactions among the particles. Furthermore, it is expected that the microscopical spatial structure of a gas in equilibrium corresponds to the most probable one according to the principles of the statistical mechanics.

The present work is based on the assumption that the microscopical structure of a gas can be described by some class of partition of space among the particles, and that the most probable partition can be inferred using the formalism of the classical statistical mechanics. The region assigned to a particle, denoted $v$, will be called the available volume of the particle because it gives a notion of the size of the particle-free space which surrounds it 31. We have mentioned that the division of the space occupied by the gas is not unique and, therefore, an exact definition of $v$ is non-trivial in advance. However, it is possible to establish a statistical formalism of the space partition in a gas without an explicit description of the volume $v$. The application of the theory to the ideal gas allows us to identify the available volume in this simple system. This solution will provide a reference for understanding more complex systems composed by interacting particles.

\section{GAS DESCRIPTION}

We consider a gas composed of $N$ particles in a $D$ dimensional container of size $V$. The region $V$ is a segment, a surface or a volume for $D=1,2$ or 3 , respectively, although it will be called generically a volume. At low densities the state of the gas can be described using one-particle states. Since we are specially interested in the configurational state of the gas, the translational description is here omitted and its well-known contribution to the fluid free-energy will be added later.

The physical space occupied by the gas at a given instant is distributed among the $N$ particles. To each particle a certain volume $v$ is assigned (so-called the available volume, see $\llbracket$ of the total space $V$. The statistical study of space partition can be performed based only on the constrains of normalization (there is a volume $v$ per particle) and space-filling (the sum of all of them equals the total gas volume), without an exact specification of $v$ in advance. We represent the configurational state of the gas by an occupation number distribution $N_{v}$, where $N_{v} d v$ is the number of particles with available volumes between $v$ and $v+d v$. The physically allowed distributions must satisfy the following conditions

$$
\begin{gathered}
N=\int_{0}^{V} N_{v} d v, \\
V=\int_{0}^{V} v N_{v} d v .
\end{gathered}
$$

Configurational entropy. The configurational entropy of the gas is represented by the Boltzmann-Planck expression $S_{\text {conf }}=k \ln W$, where $k$ is Boltzmann's constant and $W$ the number of configurational microstates of the gas which reproduce a specific state $N_{v}$. Following the usual procedure, we subdivide the total volume into small cells which contain fractions $d w$ of neighboring states. In order to evaluate $d w$ we turn the attention to well-known results of statistical mechanics. There are $d \mathbf{l}=V d^{D} p / h^{D}$ possible states for a particle confined in the volume $V$ having a momentum $\mathbf{p}$ within a momentum volume $d^{D} p$ ( $h$ is Planck's constant). The number of states that corresponds to a physical volume $d^{D} x$ is

$$
\text { state number in }\left(d^{D} x, d^{D} p\right)=\frac{d^{D} x}{V} d \mathbf{l} \text {. }
$$


This result suggests that the fraction of configurational states of a particle having an available volume between $v$ and $v+d v$ (and any momentum) is proportional to $d v$ with a proportionality factor $V^{-1}$.

Thus, a cell has a fraction of states $d v / V$ and contains $N_{v} d v$ particles for a given occupation set $N_{v}$. It is readily shown by well-known methods in statistical mechanics that

$$
W=N ! \prod_{v}^{*} \frac{(d v / V)^{N_{v} d v}}{\left(N_{v} d v\right) !}=\frac{N !}{V^{N}} \prod_{v}^{*} \frac{(d v)^{N_{v} d v}}{\left(N_{v} d v\right) !} .
$$

The asterisk means that we must multiply over groups of single particle states instead of all states, according to the cell division. In the last term on r.h.s. of Eq. (4), $V$ was removed from the product taking into account that $\sum_{v}^{*} N_{v} d v=N$. We stress that the assignation of a volume $v$ to each particle does not imply a knowledge of the particle positions in the physical space or that the particles should be at specific sites. In this sense, the present configurational description of a system of particles can be applied to different matter structures (gases, liquids and solids).

The calculation of $S_{\text {conf }}$ is straightforward. Using Stirling's theorem in the form $m ! \approx(m / e)^{m}$, we obtain

$$
S_{\text {conf }}=-k \int_{0}^{V} N_{v} \ln \left(\frac{N_{v} V}{N}\right) d v
$$

where we have returned to an enumeration over all oneparticle states instead of groups of states and replaced a sum over $v$ by an integral.

The Helmholtz energy. The free energy of the gas is the sum of three terms

$$
F=F_{\text {trans }}+F_{\text {conf }}+U_{\text {int }} .
$$

$F_{\text {conf }}=-T S_{\text {conf }}$ is the configurational free-energy (being $T$ the temperature of the gas) and $F_{\text {trans }}$ the translational free-energy, which in equilibrium is given by,

$$
F_{\text {trans }}=\frac{N}{\beta} \ln \left(\frac{\lambda^{D}}{V}\right),
$$

where $\beta=1 /(k T)$ and $\lambda=\left(2 \pi \hbar^{2} \beta / m\right)^{1 / 2}$ is the thermal de Broglie wavelength $(\hbar=h /(2 \pi)$ and $m$ is the mass of one particle). $U_{\text {int }}$ is the sum of all energy contributions originated from the interactions among the particles. It is calculated as follows.

Interaction energy. The average of the sum of twoparticle interactions may be expressed as

$$
U_{\text {int }}=\frac{N}{2 V} \int_{0}^{V} N_{v} \phi_{v} d v
$$

where

$$
\phi_{v}=\int_{0}^{[V]} u_{v}(\omega) g_{v}(\omega) d \omega
$$

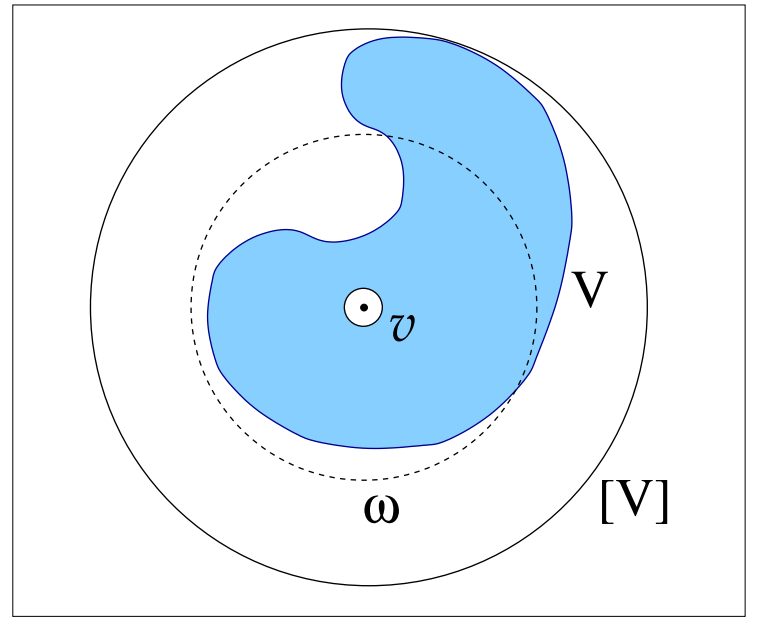

FIG. 2: Volumes involved in the evaluation of interaction energy between a reference particle (central point) with available volume $v$ (schematically represented by a circle) and the rest of the gas contained in $V$ (shaded area). The volume $\omega$ centred in the chosen particle is used to evaluated the interaction of this with the rest of the gas. $[V]$ is the minimum volume $\omega$ which encloses the gas.

Here $u_{v}(\omega)$ is the pair interaction potential and $g_{v}(\omega)$ the pair distribution function (p.d.f.) corresponding to particles with available volume $v$. Both functions are expressed as a function of the volume $\omega$ enclosed by the interaction distance $r$.

Eqs. (8 9) arise from considering the interactions between a particle having an available volume $v$ and $n g_{v}(\omega) d \omega$ particles $(n=N / V)$ located between the surfaces of spherical volumes $\omega$ and $\omega+d \omega$ centred on the chosen particle. The integration over $\omega$ takes into account all possible interactions of this particle with the remaining ones in $V$. This evaluation is repeated (integration over $v$ ) for considering the interaction of each particle with the remaining gas. A factor of $1 / 2$ avoids the double count of binary interactions. The upper limit $[V]$ of integration in Eq. (9) depends on the position of the reference particle within the gas volume and must be chosen as such that it completely covers $V$ (see Fig. 2). However, it is assumed in Eq. (9) that $\phi_{v}$ is independent from the particle position in $V$ [since $u_{v}(\omega) \rightarrow 0$ rapidly as $\omega \rightarrow \infty$, for typical fluids]. We must adopt the convention that portions of $\omega$ outside the volume $V$ do not contribute to the integral. This prescription is applied to all integrals over $\omega$ of the present paper. Since $n g_{v}(\omega)$ integrated over $V$ gives $N-1$ (i.e., the total number of particles around a chosen particle with available volume $v)$, the p.d.f. $g_{v}(\omega)$ must verify

$$
\int_{0}^{[V]} g_{v}(\omega) d \omega=V-\frac{1}{n} .
$$

The dependence of the pair particle potential with the available volume assumed in Eq. (9) is not superfluous. It is well-known that many-body effects can modify the 
interaction between two particles. For example, $N$-body effects commonly soften the repulsive part of the twobody interactions in atomic and molecular fluids. In liquid hydrogen, the repulsion between two $\mathrm{H}_{2}$ molecule is modified when a third molecule (or more) is placed nearby, because this induces changes in the electronic clouds of the particles 32]. A softening effect on pair potentials is also observed in liquid helium at high density 33. The confinement effects in the forces experimented by the particles can be implicitly included in effective pair potentials (or so-called pseudopotentials) 34], for example with the adoption of a density-dependent pair potential 33. Alternatively, many-body effects in binary interactions could be represented in the present framework with a dependence of the interaction potential $\left(u_{v}\right)$ of a particle on the free space around it. It is worth noting that if the pair potential is independent from $v$, the interaction energy $\left(\phi_{v}\right)$ of a particle can be even a function of the available volume throught the p.d.f. $\left(g_{v}\right)$.

When the pair potential is independent from the available volume of the reference particle, Eqs. (89) are reduced to

$$
U_{i n t}=\frac{N^{2}}{2 V} \int_{0}^{[V]} u(\omega) g(\omega) d \omega
$$

where

$$
g(\omega) \equiv\left\langle g_{v}(\omega)\right\rangle=\frac{1}{N} \int_{0}^{V} g_{v}(\omega) N_{v} d v
$$

$g(\omega) d \omega$ is the probability to find a particle at a distance between $r$ and $r+d r$ ( $\omega$ being the size of a $D$-sphere of radius $r$ ) from another fixed particle regardless of its available volume. Therefore, this p.d.f., written in terms of the interparticle distance $r$, coincides with the radial distribution function $g(r)$ typically used in theories of liquids and plasmas (e.g., 35]). Consequently, Eq. (11) is the standard expression of the interaction energy in a fluid. Therefore, the conventional form of the total potential energy of a one-component, classical fluid is automaticaly recovered from Eqs. (8.9), as well as the p.d.f. $g(r)$ is recovered from Eq. (12) using the more detailed p.d.f. $g_{v}(\omega)$.

\section{EQUILIBRIUM STATES}

The three contributions to the r.h.s. of Eq. (6) may be joined together into a unique expression. The total free-energy of the gas in kinetic equilibrium now reads

$$
F=\int_{0}^{V} N_{v}\left[\frac{1}{\beta} \ln \left(\frac{N_{v} \lambda^{D}}{N}\right)+\frac{N \phi_{v}}{2 V}\right] d v .
$$

At low densities, high-order correlations in the particle distribution may be neglected so that $g_{v}(\omega)$ and (therefore) $\phi_{v}$ are independent from $N_{v}$. The exact form of $g_{v}(\omega)$ depends on the considered gas. We shall return to this point later (Secs. $\nabla$ and $\nabla \mathbf{D}$ ).
The equilibrium of the particle distribution is determined by the usual method. For low density gases, the minimization of $F$ with the subsidiary conditions [Eqs. [12)] yields

$\int_{0}^{V} \delta N_{v}\left[\frac{1}{\beta} \ln \frac{N_{v} \lambda^{D}}{N}+\frac{N \phi_{v}}{2 V}+\frac{U_{i n t}}{N}-\alpha+\gamma v\right] d v=0$,

where $\alpha$ and $\gamma$ are the Lagrange's parameters associated with conservations of the particle number and the volume. Since Eq. (14) must be verified for arbitrary functional variations $\left\{\delta N_{v}\right\}$, we conclude that the equilibrium density is

$$
N_{v}=\frac{N}{\lambda^{D}} \exp \left[-\beta\left(\gamma v+\frac{N \phi_{v}}{2 V}+\frac{U_{i n t}}{N}-\alpha\right)\right] .
$$

Substitution of this expression in Eq. (13) gives the equilibrium Helmholtz energy, which can be easily written in the form of a Euler-like equation

$$
F=-\gamma V+\alpha N-U_{\text {int }} .
$$

The parameters $\gamma$ and $\alpha$ are closely related to the pressure $P$ and the chemical potential $\mu$. In order to demonstrate it, we first derive two useful identities. By introducing Eq. (15) in Eq. (11) we find that,

$$
\lambda^{D}=\int_{0}^{V} A d v
$$

where $A$ is the exponential term of $N_{v}$ in Eq. (15). Besides, since the current independent variables are the temperature, the particle number and the volume, we formally have $\alpha=\alpha(T, N, V)$ and $\gamma=\gamma(T, N, V)$. Differentiating expression (17) with respect to $N$ (using the Leibniz's theorem [36]) we obtain

$$
\begin{array}{r}
\left(\partial_{N} \alpha-\frac{\partial_{N} U_{i n t}}{N}+\frac{U_{i n t}}{N^{2}}\right) \int_{0}^{V} A d v \\
-\partial_{N} \gamma \int_{0}^{V} v A d v-\frac{1}{2 V} \int_{0}^{V} \phi_{v} A d v=0 .
\end{array}
$$

Integrals in Eq. (18) may be written in terms of $N, V$ and $U_{\text {int }}$. A little algebra leads to the first identity,

$$
N \partial_{N} \alpha-V \partial_{N} \gamma-\partial_{N} U_{\text {int }}=0 .
$$

The second one follows from the differentiation of Eq. (17) with respect to $V$. The result is

$$
\begin{array}{r}
N \partial_{V} \alpha-V \partial_{V} \gamma-\partial_{V} U_{i n t}+\frac{U_{i n t}}{V} \\
-\frac{N}{2 V} \int_{0}^{V} \partial_{V} \phi_{v} N_{v} d v=0 .
\end{array}
$$

Here a term proportional to $N_{v=V}$ has been dropped since it tends to zero in the thermodynamic limit ( $\mathrm{T}$ limit, $N, V \rightarrow \infty$ keeping $N / V$ constant). Typically, the interaction potential $u_{v}$ does not depend on $V$, so the 
integral term in Eq. (20) may also be neglected in the T-limit. Then, the second identity takes the form of

$$
N \partial_{V} \alpha-V \partial_{V} \gamma-\partial_{V} U_{i n t}+\frac{U_{i n t}}{V}=0 .
$$

From the usual thermodynamic relations and Eq. 16 it follows that

$$
\begin{gathered}
\mu \equiv \partial_{N} F=\alpha+N \partial_{N} \alpha-V \partial_{N} \gamma-\partial_{N} U_{i n t}, \\
P \equiv-\partial_{V} F=\gamma+V \partial_{V} \gamma-N \partial_{V} \alpha+\partial_{V} U_{i n t} .
\end{gathered}
$$

With the help of identities (19) and (21), we find that,

$$
\begin{gathered}
\mu=\alpha, \\
P=\gamma+\frac{U_{i n t}}{V} .
\end{gathered}
$$

Therefore, $\alpha$ is identified with the chemical potential, while $\gamma$ equals to the pressure minus the density of interaction energy. Substituting Eqs. (24) and (25) into Eq. (16) yields

$$
F=-P V+\mu N,
$$

according to the Euler's relation [37].

The program for the application of the present theory is as follows. Given the pair potential of a fluid, the evaluation of Eqs. (11) and (2) with the equilibrium distribution [Eq. [15)], yields the chemical potential $\mu$ and $\gamma$ in terms of $T, N$, and $V$. Substitution of these solutions and $U_{\text {int }}$ [which is a function of $N$ and $V$, see Eq. (8)] into Eq. (16) gives the characteristic function $F=F(T, N, V)$. As it is well-known, the availability of the thermodynamic potential $F$ in terms of the independent variables $T, N$ and $V$, provides us with the full thermodynamics description of the system.

\section{PERFECT GAS}

For a gas composed of non-interacting particles $u_{v}(\omega)=\phi_{v}=U_{\text {int }} \equiv 0$. The chemical potential $\mu, \gamma$ and the space distribution are determined by substituting Eq. (15) into the conditions given in Eqs. (11) and (2). Hence we obtain,

$$
\begin{gathered}
\mu=k T \ln \left(\frac{\lambda^{D} \beta \gamma}{1-e^{-\beta \gamma V}}\right), \\
\beta \gamma=\frac{N}{V}\left[\frac{1-(1+\beta \gamma V) e^{-\beta \gamma V}}{1-e^{-\beta \gamma V}}\right], \\
N_{v}=N \frac{\beta \gamma e^{-\beta \gamma v}}{1-e^{-\beta \gamma V}} .
\end{gathered}
$$

Since there is no interaction energy in the gas, $P=\gamma$. In the T-limit, equations of state (27) and (28) recover their usual forms $(\beta \gamma \rightarrow n)$

$$
\begin{aligned}
& \mu=k T \ln \left(n \lambda^{D}\right), \\
& P=n k T,
\end{aligned}
$$

while the spatial distribution of particles is described by

$$
n_{v} \equiv \frac{N_{v}}{V}=n^{2} \exp (-n v) .
$$

Henceforth, $n_{v}$ is called double density because its units. From Eqs. (26) and (30), the free energy takes the expected form $F=N k T\left[\ln \left(n \lambda^{D}\right)-1\right]$.

\section{A. Identification of $v$}

We have noted in $\amalg$ that the elucidation of the available volume per particle is non-trivial and difficult to find previous to the application of the equilibrium theory. The present application to a perfect gas shows that the volume $V$ is distributed among the particles following a decreasing exponential law [Eqs. (29) and (31)]. Most particles have available volumes going to zero, while few particles have a larger self space. This behavior is the same for all dimensions $D=1,2$ and 3 .

It is instructive to compare this result with the size distribution of Voronoï regions associated to systems of noninteracting particles. The size distribution of Voronoï tesselations in one dimension is given exactly by a $\Gamma$ distribution 22]

$$
n_{v}^{(\text {Voronoi })}=\frac{c n^{2}}{\Gamma(c)}(c n v)^{(c-1)} \exp (-c n v),
$$

where $c=2 D$ and $\Gamma$ denotes the usual gamma function. For higher dimensions $(D>1)$ there are no exact results, although the size distributions can be even fitted by Eq. (32) for two and three dimensions [22]. As we mentioned in Sec. III the Voronoï regions consist of all points closest to the particle chosen and, therefore, represent segments, poligons and polyhedra for $D=1,2$ and 3, respectively. Althought the Voronoï space partition could be considered a good cantidate for the equilibrium distribution $N_{v}$ derived in Sec. IV [Eq. (15]], by comparison of Eqs. (31) and (32), it is clear that the available volume per particle adopted by the thermostatistical theory applied to perfect gases is not a Voronoï region.

However, there is a simple interpretation of $v$ for a perfect gas, as we now observe. We notice that the distribution $n_{v}$ obtained for a system of non-interacting particles is equivalent (after a normalization) to the Hertz or nearest-neighbor distribution function $H(r)$ associated to such systems [38]. In fact, the number $n_{v} d v$ of particles with available volume between $v$ and $v+d v$ given by Eq. (31) is just the number $H(r) d r$ of particles which have the center of the nearest particle lying at a distance between 


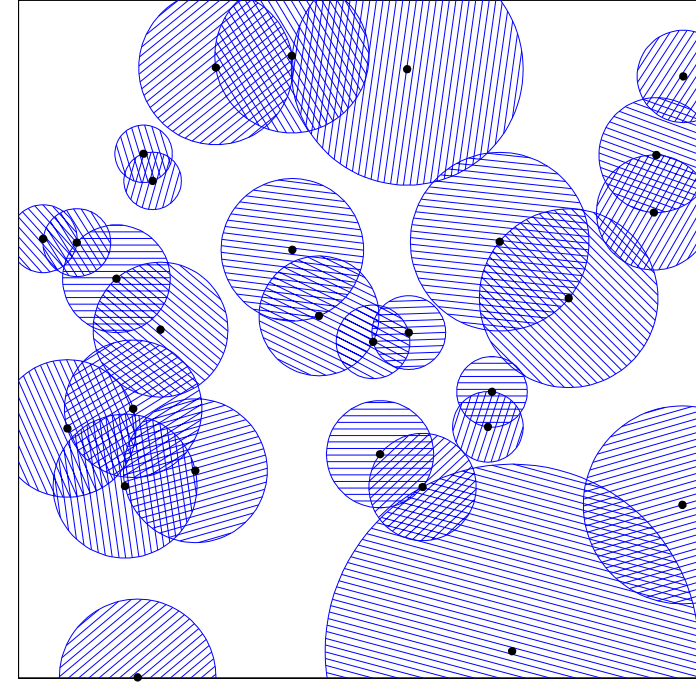

FIG. 3: Typical configuration of uncorrelated points in two dimensions. Shaded circles denote the available volumes of the objects (see text).

$r$ and $r+d r$, i.e., between the surfaces of $D$-dimensional spheres of sizes $v(r)=v$ and $v(r+d r)=v+d v$ centred on the reference particle [see Eq. [38]]. This analogy suggests that the volume $v$ of a particle in a perfect gas in equilibrium can be directly related to its distance to the nearest neighbor. Specifically, for a system of noninteracting bodies we propose the following identification of the available volume per particle,

$$
\begin{aligned}
v \equiv & D \text {-dimensional sphere of radius given by the } \\
& \text { distance between the centers of the reference } \\
& \text { particle and its nearest neighbor. }
\end{aligned}
$$

Fig. 3 illustrates the available volumes associated to a two-dimentional collection of Poisson points. One notices that the proposal (33) does not establish an ownership connection between points of the space and each particle, so that the space is not divided into distinct, nonoverlapping regions. Nevertheless, we have not found any conflict between this identification of $v$ and the theory. On the contrary, this proposal for $v$ contains two useful advantages. First, as we shall show below, the present identification of $v$ leads to a simple and accurate representation of the p.d.f. $g_{v}$. Second, $v$ gives an adequated notion of the free-particle space surrounding each particle and provides an useful tool for evaluating the perturbations experimented by atoms and molecules in diluted real gases. We will return to this point in

Fig. 4 shows a direct test of Eq. (31) and the identification (33) by computer simulation. Systems of $N=10^{5}$ uncorrelated points were simulated in unitary spaces of one, two and three dimensions using a standard algorithm [39]. The exponential decreasing behavior of the size distribution of available volumes is clearly reproduced by the numerical experiments. As a reference, one
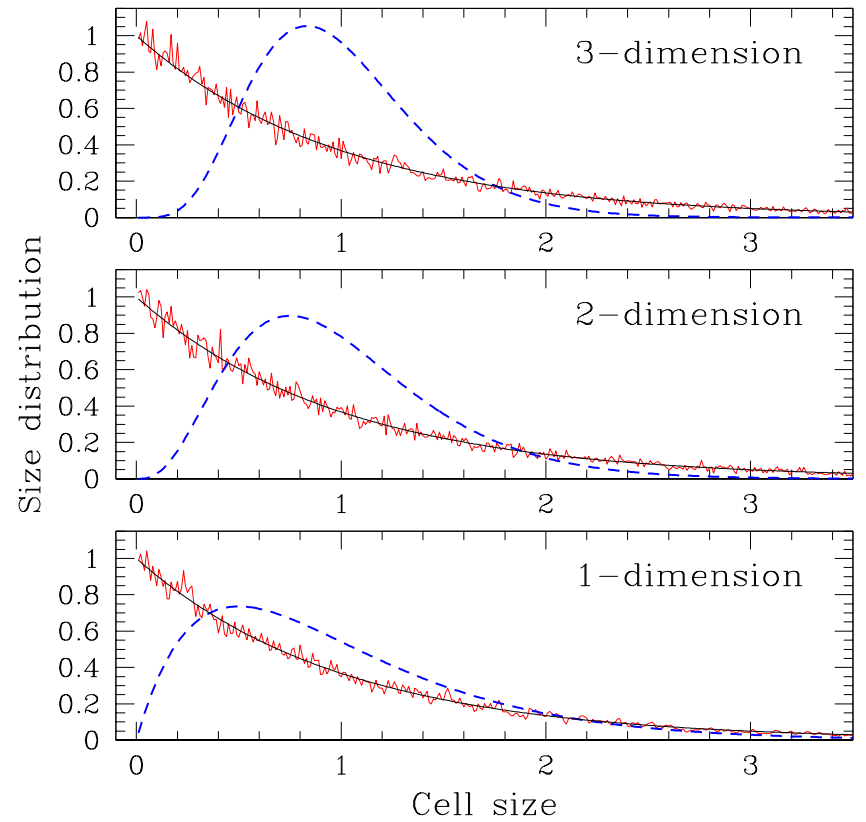

FIG. 4: Results obtained from a simulation with $5 \times 10^{4}$ Poissonian points in one, two and three dimensions. Exponential smooth curves correspond to the theoretical prediction [Eq. [31]]. The Voronoï statistics given by Eq. (32) is shown by comparison (dashed lines).

can observe in the figure the notably different laws followed by the Voronoï's space partitions (dashed lines).

\section{B. Pair distributions}

From the identification (33), the evaluation of the p.d.f. $g_{v}(\omega)$ associated to particles with available volume $v$ is immediate. We notice that $v$ gives explicitly the distance to the first neighbor, which has a contribution $g_{v}^{(1)}$ to $g_{v}$. Since $g_{v}^{(1)}(\omega) d \omega$ represents the probability of finding the nearest neighbor between the surfaces of spheres $\omega$ and $\omega+d \omega$, one find that $n \int_{0}^{\omega} g_{v}^{(1)}\left(\omega^{\prime}\right) d \omega^{\prime}$ must be zero for $\omega<v$ and one otherwise. Therefore,

$$
g_{v}^{(1)}(\omega)=\frac{1}{n} \delta(\omega-v),
$$

where $\delta(\omega-v)$ is the Dirac delta function. On the other hand, $v$ does not give any information about the positions of the remaining particles in the gas except that they are outside of a spherical volume $v$ centred on the reference particle. Besides, these neighbors are uncorrelated with the reference particle. Thus, their total contribution to $g_{v}$ must be one for $\omega>v$ and zero in the contrary case, which constitutes a Heaviside step function $\Theta(\omega-v)$. Therefore, the p.d.f. of reference particles with available volume $v$ can be expressed in the form

$$
g_{v}(\omega)=\frac{1}{n} \delta(\omega-v)+\Theta(\omega-v) .
$$


Fig. 5 confirms our identification of $g_{v}(\omega)$. It is also worthwhile to notice that Eq. (35) reproduces the expected value of the averaged p.d.f. [Eq. (12)] for a set of non-interacting particles,

$$
g(\omega)=\int_{0}^{\infty} g_{v}(\omega) n e^{-n v} d v=e^{-n \omega}+1-e^{-n \omega}=1,
$$

which expresses that the positions of the particles are completly uncorrelated and, therefore, the probability density of finding neighbors (without any specification of the available volume of the reference particle) is uniform and equals unity. We notice also that $g^{(1)}(\omega)=$ $\left\langle g_{v}^{(1)}(\omega)\right\rangle=e^{-n \omega}$ represents the first neighbor distribution for random configurations of non-interacting points. The nearest-neighbor distribution function $H(r)$, which was introduced in $\$ \nabla \mathrm{D}$ can be calculated from the relation

$$
H(r)=n \frac{d \omega(r)}{d r} g^{(1)}[\omega(r)] .
$$

Its evaluation reproduces the well-known results of a perfect gas [40],

$$
H(r)=n \frac{d \omega(r)}{d r} \exp [-n \omega(r)] .
$$

For $D=3$, it is a classical result derived by Hertz 41.

In closing this section, it is worth stressing that all termodynamics and spatial statistical, well-known results of classical perfect gases in one, two and three dimensions, are self-consistently derived from the theory developed in Secs. III and IV The identification of the available volume given in (33) plays a central role in the present application of the theory, and so we believe that it should be regarded as the most plausible interpretation of $v$ for systems of non-interacting particles.

\section{GASES OF HARD ELASTIC PARTICLES}

Systems formed by hard bodies constitute simple fluid models in which impenetrable particles interact solely via hard-core repulsions. These models have played a major role in the liquid state theory, as the behavior of dense fluids is dominated by the exclude-volume effects associated with the repulsive forces of its constituents. The main advantage of these models is the simplicity of the pair potential, which can be defined by

$$
u_{v}(\omega)= \begin{cases}\infty, & \omega \leq a \\ 0, & \omega>a\end{cases}
$$

where $a$ is the size of a $D$-sphere of radius $d, d$ being the diameter of a particle. The aim of this section is to apply the theory to hard-particle systems, which can provide a guidance for the treatment of more realistic fluids. We concentrate on the limit of low densities, although the results derived for $D=1$ will be valid at all densities.

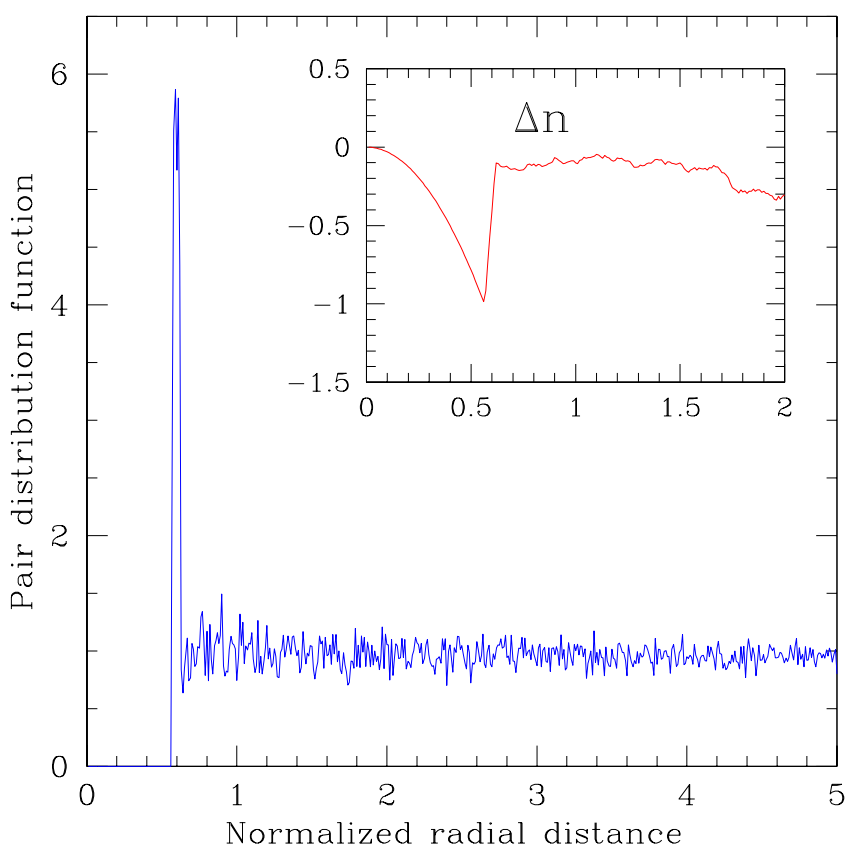

FIG. 5: Pair distribution function $g_{v}$ for particles with available volume $v=(1.1 \pm 0.1) n^{-1}$ in a two-dimension ideal gas as a function of the dimensionless distance $x=r \sqrt{n}$ (units of the mean distance between particles). The peack around $x=0.59$ is the contribution of the nearest neighbor. The results are for $N=10000$ particles randomly distributed in a unitary square surface (662 particles were selected with the chosen $v$ ). The insert graph shows $\Delta n=\int_{0}^{x}\left(g_{v}-1\right) \pi x d x$, i.e., the difference between the actual particle number within a radius $x$ with respect to that one corresponding to a (microscopical) uniform particle density. The unitary jump (within the numerical errors) in $x=0.59$ confirms that the peak of $g_{v}$ corresponds to one particle (the nearest neighbor).

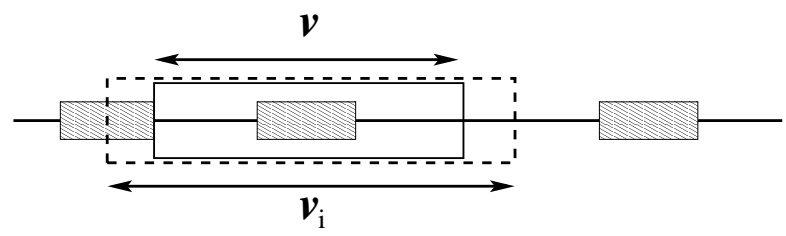

FIG. 6: Schematic representation of the available volume $v$ of a hard rod (central shaded rectangle) in presence of its nearest neighbor. The volume $v_{i}$ is the ("ideal") available volume of the central body if the bodies are fully penetrable (non-interacting), according to the interpretation (33).

As a consequence of the hard-core interaction, the identification (33) of the available volume of a particle is not valid for the present fluid. In fact, we notice that the space occupied by a particle is inaccesible to the other ones, therefore this region must be a part of the available volume of the particle not shared with the others. Consequently, the space occupied by neighbor particles yields a reduction of the available volume of a particle with respect to that $\left(v_{i}\right)$ calculated for fully penetrable 


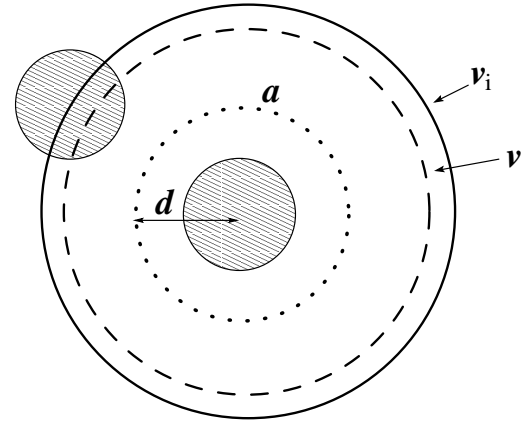

FIG. 7: Same as Fig. [6] but for $D=2$. The exclusion region $a$ (circle closed by dotted points) is a forbidden region to the particle center of neighbors. The available volume of a hard particle $(v)$ is lower than the perfect-gas value $\left(v_{i}\right)$ (see text).

(non-interacting) objects, so that $v<v_{i}$. As an ansatz, we propose that the available volume of a hard $D$-sphere is given by

$$
v=v_{i}-a^{*},
$$

where $a^{*}$ is the reduction of the available volume of a hard particle with respect to the perfect-gas value (33). As we shall show later, the value of $a^{*}$ can be evaluated using the virial theorem.

On the other hand, one expects that the p.d.f. $g_{v}$ of a perfect gas [Eq. (35)] is a good approximation to the p.d.f. of non-ideal fluids at very low densities. If we take into account the relation (40), the p.d.f. of a diluted hard-body fluid can be written as

$$
g_{v}(\omega)=\frac{1}{n} \delta\left(\omega-v_{i}\right)+\Theta\left(\omega-v_{i}\right) .
$$

From Eqs. (9), (39) and (41), we find the mean potential energy of a particle with available volume $v$,

$$
\phi_{v}= \begin{cases}\infty, & v \leq b \\ 0, & v>b\end{cases}
$$

where

$$
b=a-a^{*} .
$$

From Eq. (15) then results that $N_{v}$ is zero for $v \leq b$ and has an exponential dependence on the available volume for $v>b$. Clearly $U_{i n t}=0$ and then $P=\gamma$. At the T-limit, evaluations of Eqs. (12) yield

$$
\begin{gathered}
\mu=k T\left[\frac{n b}{1-n b}+\ln \left(\frac{n \lambda^{D}}{1-n b}\right)\right], \\
\gamma=\frac{n k T}{1-n b}, \\
n_{v}=\Theta(v-b)\left(\frac{n^{2}}{1-n b}\right) \exp \left[-\frac{n(v-b)}{1-n b}\right] .
\end{gathered}
$$

TABLE I: Quantities used in the study of $D$-dimensional hard-body fluids. $\omega(r)$, size of a $D$-sphere of radius $r ; \sigma$, volume of a hard-body of diameter $d ; \eta$, packing fraction; $a$, exclusion sphere of the repulsive interaction; $a^{*}$, reduction of the available volume per hard-body; $b=a-a^{*}$ (see text).

\begin{tabular}{rrrrrrr}
\hline \hline & $\omega(r)$ & $\sigma$ & $\eta$ & $a$ & $a^{*}$ & $b$ \\
\hline$D=1$ & $2 r$ & $d$ & $n \sigma$ & $2 \sigma$ & $\sigma$ & $\sigma$ \\
$D=2$ & $\pi r^{2}$ & $\pi d^{2} / 4$ & $n \sigma$ & $4 \sigma$ & $2 \sigma$ & $2 \sigma$ \\
$D=3$ & $4 \pi r^{3} / 3$ & $\pi d^{3} / 6$ & $n \sigma$ & $8 \sigma$ & $4 \sigma$ & $4 \sigma$ \\
\hline \hline
\end{tabular}

The free energy is easily derived from Eqs. (16), (44) and (45), and reads

$$
F=N k T\left\{\ln \left[\left(n \lambda^{D}\right) /(1-n b)\right]-1\right\} .
$$

As we anticipated, the virial theorem may provide the value of $b$ and, thus, the elucidation of the factor $a^{*}$ at low densities. According to Eq. 45), $b$ represents the second virial coefficient in the equation of state $P=n k T(1+$ $b n+\ldots)$ (since $\gamma=P$ ) and, therefore, its value is wellknown for hard $D$-spheres. If we denote with $\sigma$ the size of a hard-particle, then $b=\sigma, 2 \sigma$ and $4 \sigma$, for $D=1$, 2 and 3 [42]. Therefore, from Eq. (43) and the known values of $a$, it follows that $a^{*}=b=a / 2$. Explicit values of interest are given in Table

The averaged nearest-neighbor p.d.f. can be calculated substituting the first term on the r.h.s. in Eq. (41) and $n_{v}$ given by Eq. (46) into Eq. (12). Thus, we obtain that,

$$
g^{(1)}(\omega)=\Theta(\omega-a)\left(\frac{1}{1-n b}\right) \exp \left[-\frac{n(\omega-a)}{1-n b}\right] .
$$

Further, the p.d.f. for all neighbors except the nearest one can be derived in similar way from the second term on the r.h.s. of Eq. (41). The result is

$$
g^{(r)}(\omega)=\Theta(\omega-a)\left\{1-\exp \left[-\frac{n(\omega-a)}{1-n b}\right]\right\} .
$$

The total averaged p.d.f. is composed by the sum of these two contributions.

Knowledge of $g^{(1)}(\omega)$ permits us to evaluate the nearest-neighbor distribution function $H(r)$ from Eq. (37). For convenience, we introduce the dimensionless distance $x=r / d$ and the packing fraction $\eta=n \sigma$, which gives the fraction of the total volume occupied by the hard bodies. Then $H(x)=d H(r)$, and we obtain

$$
\begin{array}{ll}
H(x)=\frac{2 \eta}{1-\eta} \exp \left[\frac{-2 \eta(x-1)}{1-\eta}\right], & x>1 \\
H(x)=\frac{8 \eta x}{1-2 \eta} \exp \left[\frac{-4 \eta\left(x^{2}-1\right)}{1-2 \eta}\right], & x>1 \\
H(x)=\frac{24 \eta x^{2}}{1-4 \eta} \exp \left[\frac{-8 \eta\left(x^{3}-1\right)}{1-4 \eta}\right], & x>1
\end{array}
$$

for $D=1,2$ and 3 , respectively $(H=0$ at $x \leq 1)$. 


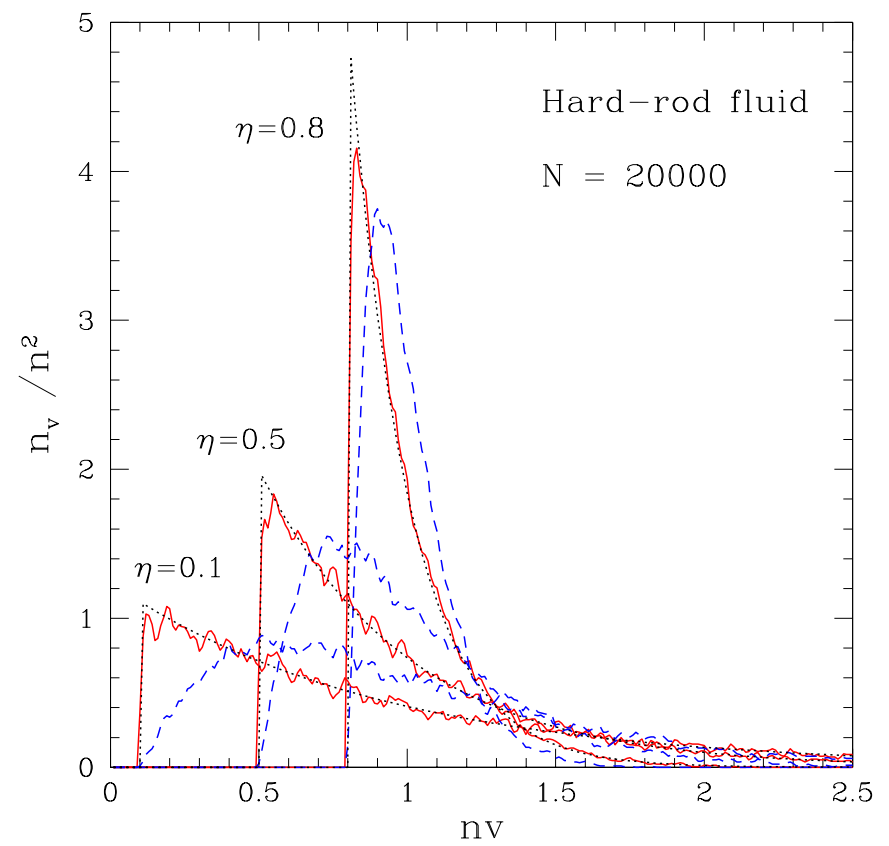

FIG. 8: Dimensionless double density of a hard-rod fluid for values of the packing fraction $\eta=n \sigma=0.1,0.5$, and 0.8 . Dotted curves correspond to the theoretical prediction [Eq. [46]) , and solid lines represent the results obtained from numerical simulations with $2 \times 10^{4}$ hard particles. The Voronoï statistics is shown by comparison (dashed lines).

\section{A. Hard-rod fluid}

The properties of a fluid of hard rods $(D=1)$ are known exactly, hence this model offers an appropriate test for our evaluations. Thus, it can be verified that the free energy given in Eq. (47) is rigorously exact for $D=1$ and, therefore, all the equations of state are correct. In particular, Eq. (45) gives the exact equation of state for hard rods as derived earlier by Tonks [43].

As we have seen in the case of a perfect gas, the present theory not only gives the thermodynamic properties of the fluid, but also provides information on its microstructure throught the doble density $n_{v}$. Fig. 8 shows $n_{v}$ for several values of the packing fraction $\eta=0.1,0.5$ and 0.8 , as obtained from Eq. (46). Due to the effect of the core repulsions [see Eq. [42)], no particle may have an available volume lower than $b$, so that the distribution $n_{v}$ is characterized by an abrupt fall in $v=b(n v=\eta$ in the figure). The distribution $n_{v}$ becomes more and more peaked as the gas concentration increases. This prediction is confirmed by numerical simulations (Fig. 8).

It may surprise us that the p.d.f. $g_{v}$ given by Eq. 410 is only an approximated expression (its form was adopted from the perfect-gas solution), while its application leads to correct results as previously described. This is due to the fact that the approximation made on $g_{v}$ rests on the second term on the r.h.s. in Eq. (41) (it ignores pair correlations between a particle and its second, third, etc., neighbors), which is not required for the determination of the double density $n_{v}$. In a linear gas only the p.d.f. of the nearest neighbor is relevant, being correctly given by the first term on the r.h.s. in Eq. (41). Thus, Eq. (48) is exact for $D=1$ and leads to the correct distribution $H(r)$ of the nearest neighbor, as given in Eq. (50). This result was derived earlier by MacDonald [4].

Finally, we recall that the present theory yields exact results for the thermodynamic and geometric structure of hard rods. It gives an important support to the identifications (33) and (40) of the available volume per particle.

\section{B. Hard disks and spheres}

Not a single exact result is known for $D>1$, but a number of numerical solutions and analytical approximations are available [46]. Inspection of the free energy [Eq. (47)] and equations of state (44) and (45) evaluated with values of $b$ given in Table $\llbracket$ reveals that our results are found to give the correct thermodynamical properties of hard discs and spheres at low densities.

In order to verify the double density $n_{v}$ given in Eq. (46), we study the nearest neighbor distribution function $H(x)$ which is derived from it. For this purpose, Eqs. (51) and (52) are contrasted with the analytical expressions derived by Torquato [40], which show an excelent agreement with Monte Carlo simulation data 47]. We use Torquato's expressions valid for densities lower than the freezing density (the freezing packing fraction is $\eta_{f} \approx 0.69$ and $\eta_{f} \approx 0.49$ for discs and spheres, respectively). Figs. 9 and 10 show that Eqs. (51] and (52) give the correct asymptotic behavior of $H(x)$ at low densities, and capture the salient features of $H(x)$ for moderate densities. The agreement between these results and Torquato's expressions is within $1 \%$ everywhere at packing fraction $\eta<0.01$ and $\eta<0.002$, for $D=2$ and $D=3$, respectively.

In closing, the low-density, asymptotic results of hard discs and spheres are correctly reproduced by the present theory. We find again a support for the identification of the available volume per particle, closely related with the distance to the nearest neighbor.

\section{Van der Waals fluid}

In the preceding subsections, we have studied the application of the theory to systems composed by particles with infinite hard cores. We shall consider now the previous model but with the inclusion of an attractive longrange contribution in the potential. Because attractive forces do not restrict the accessible space to a particle, the identification (40) of the available volume per particle (with $a^{*}$ given in Table I) should remain unaltered for this model. Instead of trying to figure out the pair 

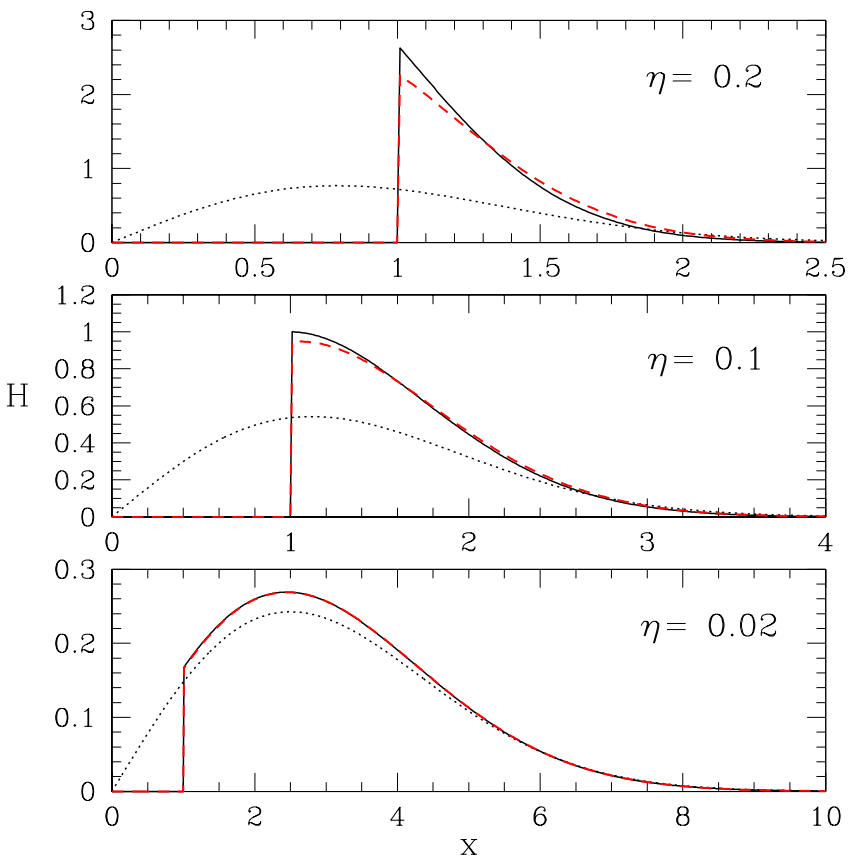

FIG. 9: Nearest neighbor function $H(x)$ of a hard-disc fluid at packing fraction $\eta=0.02,0.1$, and 0.2 , in terms of the reduced distance $x=r / d$ ( $d$, diameter of a particle). Solid curves result from Eq. (51), dashed lines correspond to accurate fits up to freezing $(\eta<0.69)$ [40], and dotted lines are results for fully penetrable particles.
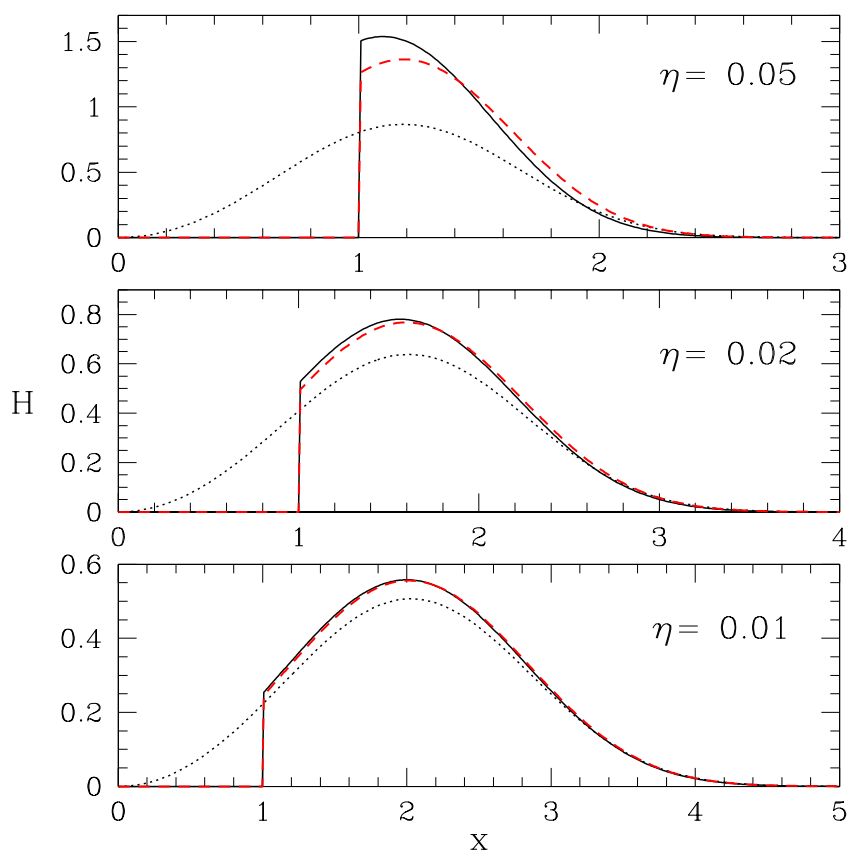

FIG. 10: Nearest neighbor function $H(x)$ of a hard-sphere fluid at packing fraction $\eta=0.01,0.02$, and 0.05 , in terms of the reduce distance $x=r / d$ ( $d$, diameter of a particle). Solid curves result from Eq. (52), dashed lines correspond to accurate fits up to freezing $(\eta<0.49)$ [40], and dotted lines are results for fully penetrable particles. potential itself, one may directly define $\phi_{v}$ as

$$
\phi_{v}= \begin{cases}\infty, & v \leq b \\ -\epsilon, & v>b\end{cases}
$$

where $\epsilon$ is a constant parameter (with units of energy times volume) and $b$ is given by Eq. (43). Eq. (53) is essential for obtaining the Van der Waals equation and is the simplest choice to test the relation (25) between the pressure and the parameter $\gamma$ when $U_{i n t}$ is non-zero.

In fact, this form for $\phi_{v}$ is based on the suitable shortrange repulsion and an attractive interaction whose range is finite but long compared to the interparticle spacing. An appropriate attractive potential with these characteristics is the general Kâc potential [48]

$$
u_{v}^{K a c}(\omega)=-\epsilon \gamma^{*} J\left(\gamma^{*} \omega\right),
$$

with $\int_{0}^{\infty} J(\omega) d \omega=1$. Here $\gamma^{*}$ represents the $D$ th power of the usual inverse-range parameter. The evaluation of the attractive-interaction energy per particle is immediate. From Eqs. (9), (41) and (54), one obtains (in the T-limit) that,

$$
\phi_{v}^{K a c}=-\frac{\epsilon}{n} \gamma^{*} J\left[\gamma^{*}\left(v+a^{*}\right)\right]-\epsilon \int_{\gamma^{*}\left(v+a^{*}\right)}^{\infty} J(\omega) \omega .
$$

In the limit $\gamma^{*} \rightarrow 0, \phi_{v}^{K a c} \rightarrow-\epsilon$ and we recover the attractive term in Eq. (53). This result shows that the attractive energy of a particle results from its simultaneous interaction with a large number of particles, where the contribution of the nearest neighbors is negligible regardless their distances to the reference one. As a consequence, $\phi_{v}^{K a c}$ is equivalent for all particles independently from their available volumes.

The interaction energy given in Eq. (53), after substitution in Eq. (15) and normalization, reproduces the double density given by Eq. (46). In the T-limit, Eqs. (11) and (2) lead to

$$
\mu=k T\left[\frac{n b}{1-n b}+\ln \left(\frac{n \lambda^{D}}{1-n b}\right)\right]-\epsilon n,
$$

while expression (45) is obtained for $\gamma$. The evaluation of Eq. (8) yields a non-zero interaction energy with density $U_{\text {int }} / V=-\epsilon n^{2} / 2$. Besides, from previous results and Eq. (16), the Helmholtz energy takes the form of

$$
F=N k T\left\{\ln \left[n \lambda^{D} /(1-n b)\right]-1\right\}-\epsilon N n / 2 .
$$

From differentation of $F$ with respect to $V$ one obtains the well-known Van der Waals equation of state,

$$
P=\frac{n k T}{1-n b}-\frac{\epsilon n^{2}}{2} .
$$

Relation (25) is thus confirmed beyond the trivial case $(P=\gamma)$ obtained for perfect and hard-body fluids. The Van der Waals model constitutes a special case where attractive forces have not influenced the value of $\gamma$, which, however, is strongly governed by the infinitely repulsive interactions. 


\section{Order in the fluid structure}

We notice that the double density offers a way to measure the order of the packing of hard particles. In fact, we can define the fluctuation of the available volume as the standard deviation

$$
\Delta v \equiv\left[\left\langle v^{2}\right\rangle-\langle v\rangle^{2}\right]^{1 / 2}
$$

where $\langle\ldots\rangle$ represents an average in the ensemble [earlier applied in Eq. (12)], namely,

$$
\left\langle f_{v}\right\rangle \equiv \frac{1}{n} \int_{0}^{\infty} f_{v} n_{v} d v
$$

$f_{v}$ being a function of the available volume per particle. Obviously, $\langle v\rangle=n^{-1}$ for all fluids [see Eq. (2)]. According to Eq. (46) for hard-body fluids, one obtains

$$
\Delta v=\langle v\rangle-b, \quad \text { (hard bodies) . }
$$

For comparison, the space fluctuation of classical perfect gases is as large as the mean volume per particle for any temperature and density [c.f. Eq. (31)],

$$
\Delta v=\langle v\rangle, \quad \text { (perfect gas) }
$$

This points out the irregular geometric structure that characterizes random collections of non-interacting objects.

The volume fluctuation $\Delta v$ given by Eq. (61), summarizes the behavior of $n_{v}$ with the density for hard rods. Large deviations of the available volumes from the mean value occur at low densities, and the hard-rod fluid has the inhomogeneity of a perfect gas in the limit $\eta \rightarrow 0$ $\left(\Delta v \rightarrow n^{-1}\right)$. As expected, the fluctuations decrease at high densities and vanish in the limit of close-packing $\eta=1$. Eq. 61 is valid for discs and spheres at low densities.

Classical methods for determining the order in an isotropic packing of hard $D$-spheres are based on geometrical and topological techniques. The former rests on the identification of peaks in the radial function $g(r)$, which is found to be unsatisfactory because, among other reasons, it is difficult to determine when the peak appears [51]. Topological methods use the so-called order or topological parameters, such as like-coordination numbers 49] and the number of faces in a cell division 14, 50. They commonly require an appreciable amount of calculation divided in two steps, first, an evaluation of configurations of the system in equilibrium states (usually based on numerical simulations), and, then, the execution of an algorithm which evaluates the cell division (typically a Voronoï tesselation).

In constrast with the previous methods, the present formalism offers, eventually, a direct and precise way to characterize the order in fluid structures in terms of the fluctuation of the available volume per particle. Thus, for example, $\Delta v=\langle v\rangle$ represents the high density fluctuations present in very diluted gases. On the contrary, uniformily ordered configurations should be characterized by space fluctuations noticeably lower than $\langle v\rangle$. Although the application of $\Delta v$ is here limited to hard particles at low densities, additional investigation could extend its use to real fluids.

\section{DILUTED HYDROGEN PLASMA}

Finally, as the main example of this work we consider a low density gas formed by hydrogen atoms in an electrically neutral background of free electrons and protons. For simplicity, we consider a strongly ionized equilibrium state where the atom-atom interaction can be ignored, and only the charge effects upon the atoms are taken into account. Each atom is considered as a polarizable particle and its available volume is approximated by the perfect-gas value (33). Our aim focuses on the description of the neutral component of the gas. We emphasize that the results obtained from this model are qualitative and illustrative only. A detailed study of a mixture of gases in equilibrium is in progress and its results will be presented elsewhere.

The equilibrium density given in Eq. (15) can be easily generalized to particles with internal states following the procedure of Secs. III and IV] The double density of atoms in the energy level $j$ and with available volume $v$ [hereafter, atoms $(j, v)]$ is given by

$$
n_{j, v}=\frac{n}{\lambda^{3}} g_{j} \exp \left[-\beta\left(\epsilon_{j}+\Delta \epsilon_{j v}+\gamma v-\mu\right)\right],
$$

where $g_{j}$ and $\epsilon_{j}$ are the multiplicity and eigenenergy of the level $j$ for an isolated atom, $n$ is the total density, and $\lambda$ and $\mu$ the thermal wavelength and chemical potential of the atomic component, respectively. The factor $\Delta \epsilon_{j v}$ can be interpreted as the energy change of a particle $(j, v)$ due to interactions with the charges. Specifically, we obtain

$$
\Delta \epsilon_{j v}=\frac{1}{2}\left(n_{e} \phi_{j v, e}+n_{p} \phi_{j v, p}\right)
$$

with

$$
\phi_{j v, c}=\int_{0}^{[V]} u_{j v, c}(\omega) g_{j v, c}(\omega) d \omega,
$$

where the subscript $c$ refers to either electrons or protons, $n_{e}$ and $n_{p}$ are the number densities of free electrons and protons, $u_{j v, c}$ is the pair potential of the interaction of an atom $(j, v)$ with a charge $c$, and $g_{j v, c}(\omega)$ represents the probability density of finding a charge $c$ in the surface of a spherical volume $\omega$ centred on an atom $(j, v)$. We have omitted in Eq. (64) the energy change of atoms in the level $j$ due to their perturbations over the charges, because it is only a small correction (proportional to the abundance of atoms) in $\Delta \epsilon_{j v}$ for the physical conditions assumed.

At large separations, charges induce a dipole moment in the hydrogen atom. The pair potential associated to polarization of an atom by either an electron or a proton can be represented by the screened Debye potential 
obtained by Redmer and Röpke 52 (in the form used in [53])

$$
u_{\text {pol }}(r)=-\frac{e^{2} \alpha_{j}}{2}\left(\frac{1+r / r_{s}}{l_{j}^{2}+r^{2}}\right)^{2} e^{-2 r / r_{s}},
$$

where $e$ is the elemental charge, $r_{s}$ the screening length, $\alpha_{j}$ the polarizability of an atom at the level $j$, and $l_{j}=$ $a_{B} j\left[\left(7 j^{2}+5\right) / 4\right]^{1 / 2}$ (with $j$ the main quantum number and $a_{B}$ the Bohr radius) its mean radius.

At short separation between an atom and a proton the Coulomb repulsion between protons prevails 53 .

$$
u_{C}(r)=\frac{e^{2}}{r}\left(1+\frac{r}{a}\right) e^{-2 r / a} .
$$

This potential includes a plasma screening function with a characteristic length $a=\frac{1}{2} a_{B} l_{j} / l_{1}$.

Due to Pauli's exclusion principle, there is a strong repulsion when the electronic wavefunctions of two atoms begin to overlap and this is often expressed as

$$
u_{12}(r)=\frac{C_{12}}{r^{12}} .
$$

We use this potential to simulate the interaction between a free electron and an atom. The coefficient $C_{12}$ is harder to evaluate. From Hindmarsh et al. 54] we adopt $C_{12}=9 \times 10^{-17} l_{j}$ erg $\mathrm{cm}^{12}$, where we consider only the wavefunction size (of characteristic radius $l_{j}$ ) of the bounded electron.

On the average, roughly half of atoms have an electron as its nearest neighbor and the other half a proton. The fraction of atoms having other atoms as nearest neighbors is negligible in a highly ionized material. Therefore, the total density of atoms can be written as

$$
n_{H}=\sum_{j} \int_{0}^{\infty} n_{j v}^{(p)} d v+\int_{0}^{\infty} n_{j v}^{(e)} d v
$$

where $n_{j v}^{(p)}$ and $n_{j v}^{(e)}$ are the double densities of atoms which have a proton or an electron as their nearest neighbor. Both densities are given by Eq. (63) (times 1/2), although they differ in the energy term $\Delta \epsilon_{j v}$. For the first and second atom sets we have,

$$
\begin{aligned}
& \Delta \epsilon_{j v}^{(p)}=\frac{1}{2}\left(u_{p}(r)+\int_{v}^{\infty}\left[n_{e} u_{e}(\omega)+n_{p} u_{p}(\omega)\right] d \omega\right), \\
& \Delta \epsilon_{j v}^{(e)}=\frac{1}{2}\left(u_{e}(r)+\int_{v}^{\infty}\left[n_{e} u_{e}(\omega)+n_{p} u_{p}(\omega)\right] d \omega\right),
\end{aligned}
$$

respectively, where $u_{p}=u_{C}+u_{p o l}$ and $u_{e}=u_{12}+u_{p o l}$ are the total atom-proton and atom-electron potentials, respectively, and $r=(3 v / 4 \pi)^{1 / 3}$. In these evaluations, Eq. (35) was adopted for the species (electrons or protons) which let the nearest neighbor of an atom $(j, v)$,
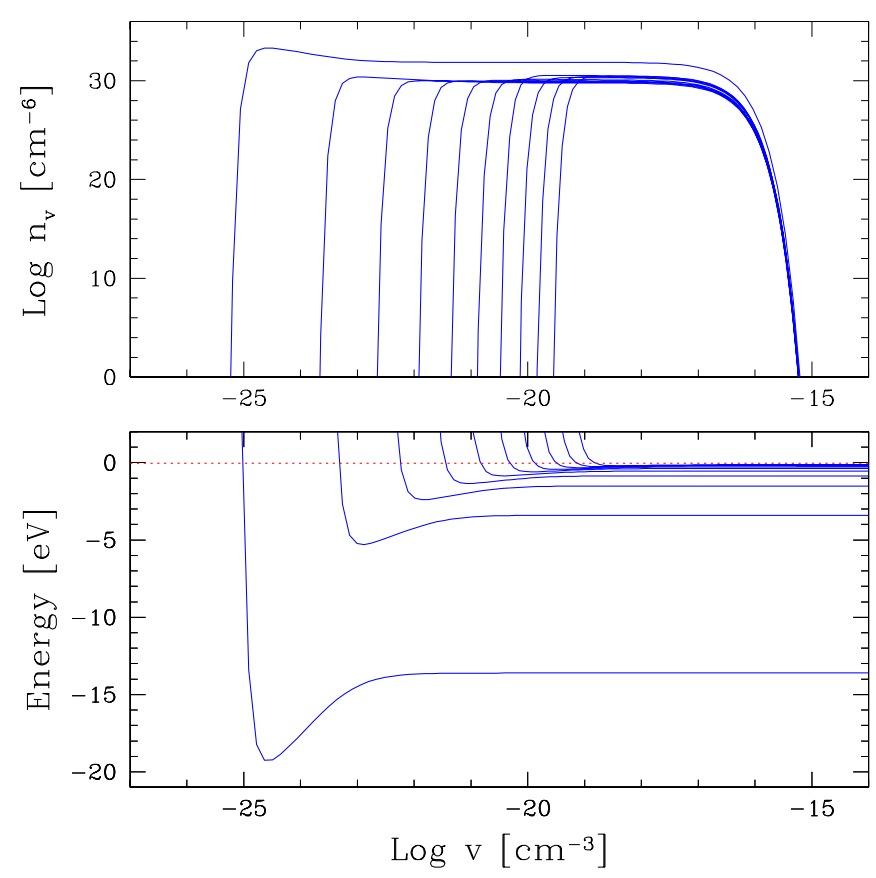

FIG. 11: Double density (above) and energy (below) curves of the first ten levels of hydrogen atoms (solid lines from left to right), which have an electron as the nearest neirghbor. The results are shown as a function of the available volume per atom, and correspond to $T=15000 \mathrm{~K}$ and mass density $\rho=$ $10^{-6} \mathrm{gr} \mathrm{cm}^{-3}$. The dotted line shows the energy continuum edge for electrons and protons.

while we approximate $g_{j v, c}(\omega)=\Theta(\omega-v)$ as the p.d.f. associated to the other perturber class.

From Eq. 63) and the division of atoms into two groups (according to the type of nearest perturber), the internal partition function of the atomic hydrogen is given by

$$
Z=\sum_{j} g_{j} e^{-\beta \epsilon_{j}} \int_{0}^{\infty} e^{-\beta \gamma v}\left(\frac{e^{-\beta \Delta \epsilon_{j v}^{(e)}}+e^{-\beta \Delta \epsilon_{j v}^{(p)}}}{2}\right) d v
$$

This partition function includes a state sum over the physical space as a direct consequence of the configurational parameter $v$ used in the gas description. When the interactions are negligible $\left(\Delta \epsilon_{j v}^{(e)}=\Delta \epsilon_{j v}^{(p)}=0\right)$, the present partition function reduces to $Z=Z_{0} / n$ where $Z_{0}=\sum_{j} g_{j} e^{-\beta \epsilon_{j}}$ is the conventional form of the internal partition function of a perfect gas.

Populations and effective internal energies of atoms as functions of the available volume per particle are calculated for a gas with a temperature $T=20000 \mathrm{~K}$ and mass density $\rho=10^{-7} \mathrm{gr} \mathrm{cm}^{-3}$. This is representative of conditions found in the atmospheres of lukewarm white dwarfs (effective temperature around $T_{\text {eff }}=15000 \mathrm{~K}$ ). Non-ideal effects on equations of state are negligible and, particularly, $\beta \gamma=n=10^{17.07} \mathrm{~cm}^{-3}$. A standard evaluation of the chemical equilibrium yields $n_{e}=n_{p}=10^{16.76}$ 
$\mathrm{cm}^{-3}$ and $n_{H}=10^{15.31} \mathrm{~cm}^{-3}$.

The effective internal energy of an atom is composed by the sum of its electronic energy $\epsilon_{j}$ (that chosen of an unperturbed atom) and the interaction energy $\Delta \epsilon_{j v}$ which condenses the plasma effects. Lower panels in Figs. 11] and [12 show the effective internal energy as a function of its available volume for the lowest atomic levels. Atoms with great volumes are, in practice, unperturbed and their internal energies are very close to those of an isolated atom. As $v$ decreases the atoms become progressively more bound due to the attractive long-range interaction with charges. This interaction has a stronger $v$ dependence for high-lying levels due to a greater shearing of the electronic wavefunction, and accordingly, a greater polarizability.

The Coulomb repulsions and Pauli's exclusion effects preclude atoms from having very low available volumes. These short-range interactions increase the effective internal energy of atoms and bound states successively merge into the continuum with decreasing the available volume. Differences between energy curves of Figs. 11 and 12 at low $v$ are due to the fact that Coulomb repulsion between protons [Eq. [67]] is appreciably softer than the electron-atom interaction [Eq. (68)]. In particular, atoms with an electron as the nearest neighbor have an abrupt increasing of interaction energy at low available volumes. Notice that linear Stark effects are not taken into account because we use energy eigenvalue $\left(\epsilon_{j}\right)$ of unperturbed particles. However, since the linear Stark effects only spread over the levels without shifting their centers of gravity, the main effect on the internal energy of the atoms is due to shifts caused by plasma polarization and short-range interactions considered in $\Delta \epsilon_{j v}$.

Density curves in Figs. 11 and 12 (upper panels) show that atoms exist only for available volumes within a certain domain, which depends on the internal state $j$. The double densities take maximum values around the minimum of the internal energy curves. The absence of particles with very low volumes $v$ is due to the fact that atoms with high internal energies are not stable. Indeed, according to the energy curves, an atom with very low available volumes is so strongly perturbed that its electron becomes free in the plasma. Atoms in high-lying bound states are more strongly affected by the presence of plasma electrons and ions, because their electrons are loosely bound. Since the Coulomb repulsion $u_{C}$ is softer than the potential $u_{12}$, atoms with a proton as the nearest neighbor may survive at lower available volume than those with an electron as the nearest neighbor. However, this difference does not have an influence on the total populations of each group of atoms.

The population of atoms in all internal states declines at high $v$ volume $\left(v \approx 10^{-15.5} \mathrm{~cm}^{-3}\right)$ because actually there are no available volumes per particle much greater than the mean volume per particle in the gas, $\langle v\rangle=n^{-1}=10^{-16.07}$. Mathematically, this is a consequence of the term $\exp (-\beta \gamma v)$ in the double density
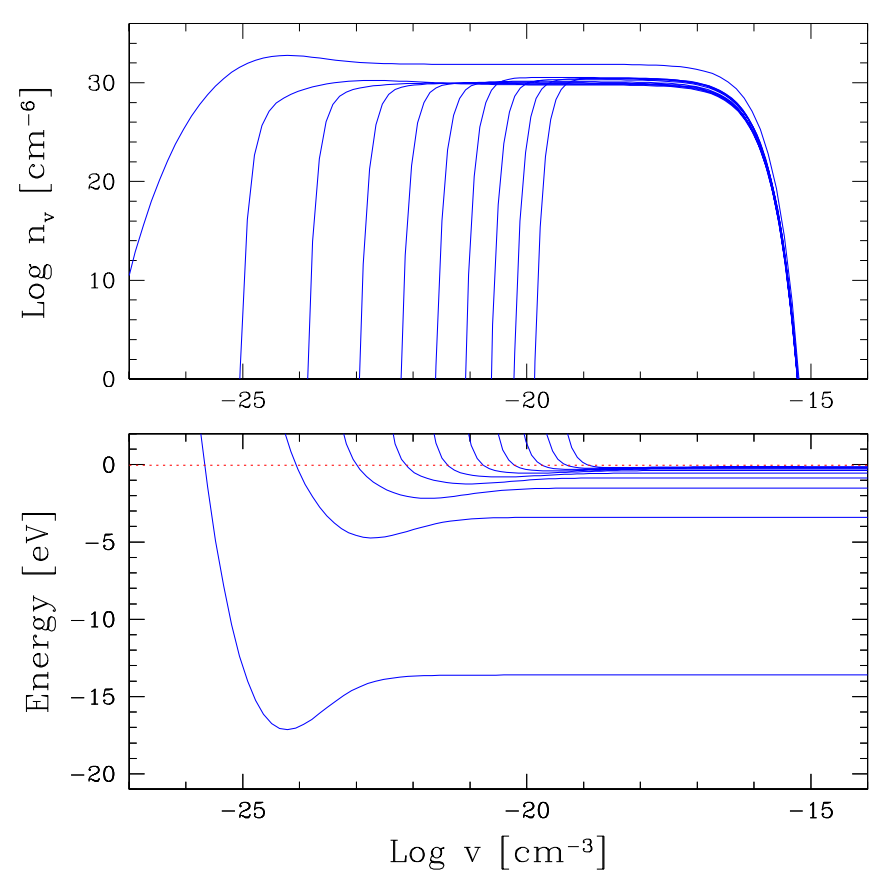

FIG. 12: Same as Fig. [11 but for atoms with a proton as the nearest neighbor.

expression [Eq. [63)]. Such factor plays the role of a probability of occupation of available volumes. Large volumes $v$ represent single-particle, configurational states with smaller occupational probability than the little ones.

Fig. 13] shows total densities (i.e., $v$-averaged) of atoms in differents internal states, together with predictions obtained for non-interacting particles. Low-energy bound states have essentianly unperturbed populations. On the contrary, atoms in high energy levels are so strongly perturbed that their equilibrium populations decrease with respect to a hypothetical gas of unperturbed atoms. Under current conditions, states with main quantum numbers larger than $\approx 30$ are not populated because the atom, in order to survive, requires a volume $v$ much larger than the mean volume per particle and the probability of existence of such space becomes very small. Notice the smooth dependence of the non-ideal effects on atomic density with the quantum main number. This contrasts with evaluations of internal partition function based on an (often used) abrupt cutoff at certain upper level. The present evaluation of internal partition function contains useful advantages derived from the free-energy minimization technique: (i) convergence of the internal partition function, (ii) incorporation of particle-interaction models in a consistent statistical mechanical way.

A variety of gas models have been developed to compute the equilibrium state of astrophysical fluids, and which include the influence of non-ideal gas effects upon the internal partition function of bound states [1, 53, [55]. However, to our knowledge, this is the first time that combined and self-consistent evaluations of atomic popu- 


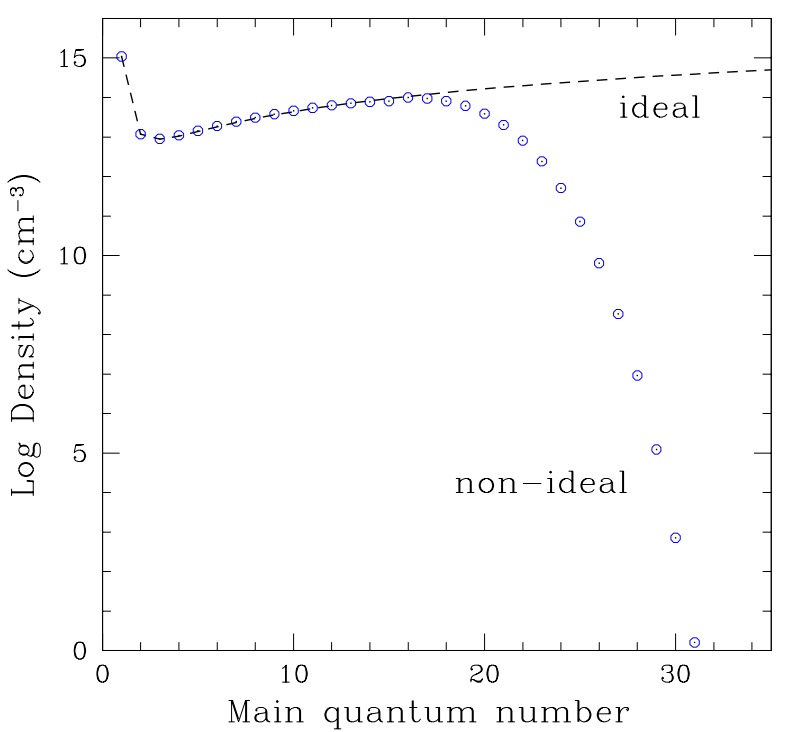

FIG. 13: Populations of atomic levels as a function of the quantum main number for $T=20000 \mathrm{~K}$ and $\log \rho=-7$. Open circles show results for atoms with an electron as the nearest neighbor, while points correspond to atoms with a proton as the n.n. (actually both atom groups have very similar populations). The dashed line shows results based on an ideal gas (i.e., for $\Delta \epsilon_{j v}^{(e)}=\Delta \epsilon_{j v}^{(p)} \equiv 0$ ).

lations and internal effects on bound states are performed differentiating groups of atoms under different plasmaperturbations. We consider this as an important advantage of the present formalism, which can be useful for more elaborated models. Notice also that the data derived from the population equation (63) could be used to set up thermodynamic functions and to obtain optical quantities. This is not however within the scope of this paper.

\section{CONCLUSIONS}

Classical statistics of gases in equilibrium has been extended taking into account a new one-particle variable which assigns a space $v$ to each particle. We call this variable the available volume per particle since it roughly represents the free space around it. We develop the theory in terms of a Helmholtz free-energy model for onecomponent gases at low densities where the particle interactions are assumed to be binary. The equilibrium state is calculated from the energy minimization with respect to the occupation number $N_{v}$ of $v$-states, subjected to particle number and volume constrains. The volumeclosure introduces a Lagrange's multiplier, here called $\gamma$, which regulates the space partition (i.e., $N_{v}$ ) and is equivalent to the pressure minus the density of interaction energy in the gas. A universal expression [Eq. (15)] gives the occupation number $N_{v}$ for dilute gases at equilibrium. When the pair potential is specified, $N_{v}$ contains both thermodynamics and structure information of the fluid. We have illustrated the application of the theory to the perfect gas and to fluids with infinitely repulsive interactions, for one, two and three dimensions. All thermodynamic and geometric-structure properties such as the nearest neihgbor distribution function of these systems have been correctly reproduced. For hard bodies in two and three dimensions, the results are valid at low densities. It was also shown that the Van der Waals model can be derived from general Kâc potentials in a straighforward way.

The identification of $v$ has been perfomed from the application of the theory to specific cases. For noninteracting particles the available volume is equivalent to the spherical volume enclosed by the distance between the centers of the particle and its nearest neighbor. In hard-body fluids, the core-repulsions reduce the value of $v$ with respect to that for fully penetrable particles. At the limit of low densities, the explicit form of $v$ for hard bodies is derived from the virial theorem. Hard particles provide a guidance for the treatment of soft-cores present in realistic fluids. In these systems, we expect that the allowed values of $v$ will be a compromise between values associated with noninteracting particles and those corresponding to hard particles. Additional work should be done on this topic. On the other hand, there is no evidence that the identification of $v$ can be modified by attractive interactions. This is reasonable because attractive forces do not restrict the accessible space to a particle.

For non-interacting particles $N_{v}$ follows an exponentially decreasing law, such that most particles (a fraction $1-e^{-1} \approx 0.63$ ) have spaces $v$ lower than the mean volume per particle $(1 / n)$. In conexion with the structure of fluids, we have showed that the double density $n_{v}$ could be a useful tool to characterize the order in fluids, particularly, through the standard deviation of the available volume $v$.

The main application of the formalism concerns with the atomic component of a partially ionized hydrogen gas. We found a statistically coherent and detailed evaluation of populations and internal energy changes of atoms as a function of the available volume per particle. Preliminary calculations show that the distinction of atoms under different plasma-perturbations emerges naturally from the theory. This finding provides sufficient confidence for further extension of the formalism to consider a complete description of gaseous mixtures, where nonideal effects on optical properties may be investigated.

\section{Acknowledgments}

Thanks are due to J. Cantó, L. García-Colín, D. Page, S. Sahal-Bréchot and J. Zorec for their help, comments and discussions on the research reported in this paper. I 
also want to thank to L. García-Colín for a careful reading of the manuscript and valuable comments, J. Zorec for their kind hospitality during me stay in the Institut
D'Astrophysique of Paris, and Jana Benda for improving the language of the manuscript.
[1] G. Fontaine, H. C. Jr. Graboske, H. M. Van Horn, ApJS, 35, 293 (1977).

[2] See, e.g., Chr. Füchtbauer, G. Joos, and O. Dinkelacker, Ann. d. Physick 71, 204 (1923); H. P. Roberton, J. M. Dewey, Phys. Rev. 31, 973 (1928); H. Margenau, Phys. Rev 40, 387 (1932); F. L. Mohler, ApJ 90, 429 (1939).

[3] H. R. Griem, Plasma Spectroscopy (McGraw-Hill, New York, 1964).

[4] P. Bergeron, F. Wesemael, G. Fontaine, ApJ 367, 253 (1991).

[5] P. Bergeron, M. T. Ruiz, S. K. Leggett, ApJS 108, 339 (1997); P. Bergeron, ApJ 558, 369 (2001).

[6] P. Bergeron, S. K. Leggett, ApJ 580, 1070 (2002).

[7] W. Däppen, L. Anderson, D. Mihalas, ApJ 319, 195 (1987).

[8] I. Hubeny, D. G. Hummer, T. Lanz, A\&A 282, 151 (1994).

[9] T. Lanz and I. Hubeny, ApJ 439, 905 (1995).

[10] R. D. Rohrmann, A. M. Serenelli, L. G. Althaus, O. G. Benvenuto, MNRAS 335, 499 (2002).

[11] D. G. Hummer and D. Mihalas, ApJ 331, 794 (1988).

[12] E. Fermi, Zs. Phys. 26, 54 (1924); H. C. Urey, ApJ 59, 1 (1924); W. J. Class, Rech. Obs. Utrecht 12 (I), 13 (1951); G. Elste and J. Jugaku, ApJ 125, 742 (1957); C. de Jager and L. Neven, Bull. Astr. Inst. Netherlands 15, 55 (1960).

[13] H. Margenau and M. Lewis, Rev. Mod. Phys. 31, 569 (1959).

[14] N. Rivier, Phil. Mag. B 52, 795 (1985).

[15] H. C. Graboske, Jr., D. J. Harwood, and F. J. Rogers, Phys. Rev. 186, 210 (1969).

[16] F. T. Lewis, Anat. Rec. 38, 341 (1928); V. V. Smoljaninov, Mathematical Models of Biological Tissues (Moscow: Nauka, 1980) (in Russian); K. J. Dormer, Fundamental Tissue Geometry for Biologists (Cambridge: Cambridge University Press, 1980).

[17] The first ecologists to represent the space that closely surrounds a plant by Voronoï polygons were G. S. Brown [New Zealand Forestry Service Research Notes 38, 1 (1965)] and R. Mead [Annals of Botany 30, 301 (1966)].

[18] M. Hasegawa, M. Tanemura, Ann. Inst. Stat. Math. 28B, 509 (1976); J. A. Byers, J. Animal Ecology 61, 759 (1992).

[19] P. A. Mulheran, Phys. Rev. E 51, R3803 (1995); T. Aste, K. Y. Szeto, W. Y. Tam, Phys. Rev. E 54, 5482 (1996); S. Hilgenfeldt, A. M. Kraynik, S. A. Koehler, H. A. Stone, Phys. Rev. Lett. 86, 2985 (2001).

[20] S. Sastry, T. M. Truskett, P. G. Debenedetti, S. Torquato, F. H. Stillinger, Mol. Phys. 95, 289 (1998); A. Vishnyakov, P. G. Debenedetti, A. V. Neimark, Phys. Rev. E 62, 538 (2000).

[21] C. J. Schrijver, H. J. Hagenaar, A. M. Title, ApJ 475, 328 (1997).

[22] T. Kiang, Z. Astrophys. 64, 433 (1966).

[23] L. Pásztor, Astronomical Data Analysis Software and Systems III, ASP C.S. 61, 253 (1994).
[24] V. Icke, R. van de Weygaert, A\&A 184, 16 (1987); F. Bernadeu, R. van de Weygaert, MNRAS 279, 693 (1996); A. Gabrielli et al., Phys. Rev. D 67, 043506 (2003).

[25] Due to the wide field of interest, there is an extensive terminology. Dirichlet regions, Wigner-Seitz cells, Thiessen figures, Voronoï-Delaunay and Bernal's polyhedra are some of the terms usually employed in the literature to refer to regions resulting of a variety of space partitions.

[26] The earliest use of Voronoï tessellations can be found in the work of G. L. Dirichlet [J. Reine Angew. Math. 40, 209 (1850)] and G. F. Voronoï [J. Reine Angew. Math. 136, 67 (1909)].

[27] A. Okabe, B. Boots, and K. Sugihara, Spatial Tesselation Concepts and Applications of Voronoï Diagrams (John Wiley and Sons, New York, 1992).

[28] M. Baus and J.-P Hansen, Physical Reports 59, 1 (1980); D. Levesque, J-J. Weis, J. Lebowitz, Journal of Stat. Phys. 100, 209 (2000).

[29] A. Gabrielli, M. Joyce, F. Sylos Labini, Phys. Rev. D 65, 083523 (2002).

[30] P. Richard, L. Oger, J.-P. Troadec, and A. Gervois, Phys. Rev. E 60, 4551 (1999); S. Sastry, T. M. Truskett, P. G. Debenedetti, S. Torquato, and F. H. Stillinger, Mol. Phys. 95, 289 (2001).

[31] It should not be confused with the so-called available space in a liquid (sometimes also termed cavity volume), which represents the region of space that is available for inserting an additional particle into the system [see, e.g., P. Debenedetti and T. M. Truskett, Fluid Phase Equilibria, 158, 549 (1999)]. Another concept commonly used in theory of liquids is the free volume, which is defined as the volume over which the center of a given particle can translate, given that the other particles are fixed [W. G. Hoover, W. T. Ashurst, and R. Grover, J. Chem. Phys. 571259 (1972)].

[32] M. Ross, F. H. Ree, and D. A. Young, J. Chem. Phys. 79, 1487 (1983).

[33] J. M. Aparicio, G. Chabrier, Phys. Rev. E 50, 4948 (1994).

[34] I. M. Torrens, Interatomic Potentials, (Academic Press, New York, 1972).

[35] S. Ichimaru, Rev. Mod. Phys. 54, 1017 (1982); J. P. Hansen and I. R. McDonald, Theory of Simple Liquids, 2nd ed (Academic, New York, 1986).

[36] M. Abramowitz, I. A. Stegun, eds., Handbook of Mathematical Functions (Dover, New York, 1965).

[37] H. B. Callen, Thermodynamics and an Introduction to Thermostatistics, 2nd ed, (John Wiley \& Sons, New York, 1985).

[38] S. Chandrasekhar, Rev. Mod. Phys. 15, 1 (1943).

[39] W. H. Press, B. P. Flannery, S. A. Teukolsky, and W. T. Vetterling, Numerical Recipes (Cambridge Univ. Press, Cambridge, 1986).

[40] S. Torquato, Phys. Rev. E 51, 3170 (1995).

[41] P. Hertz, Math. Ann. 67, 387 (1909).

[42] A. Santos, S. B. Yuste, and M. López de Haro, Mol. Phys. 
99, 1959 (2001).

[43] L. Tonks, Phys. Rev. 50, 955 (1936).

[44] J. R. MacDonald, Mol. Phys. 44, 1043 (1981). See also eq. (5.11) in [45].

[45] S. Torquato, B. Lu, and J. Rubinstein, Phys. Rev. A 41, 2059 (1990).

[46] M. Baus and J. L. Colot, Phys. Rev. A 36, 3912 (1987).

[47] S. Torquato, S. B. Lee, Physica A 167, 361 (1990).

[48] M. Kâc, G. E. Uhlenbeck, and P. C. Hemmer, J. Math. Phys. 4, 216 and 229 (1963); J. L. Lebowitz and O. Penrose, J. Math. Phys. 7, 98 (1966).

[49] J. Steinhardt, D. R. Nelson, and M. Ronchetti, Phys. Rev. B 28, 784 (1983); P. Richard, L. Oger, J.-P. Troadec, and A. Gervois, Phys. Rev. E 60, 4551 (1999).

[50] R. Jullien, P. Jund, D. Caprion, and D. Quitmann, Phys.
Rev. E 54, 6035 (1996).

[51] M. D. Rintoul and S. Torquato, J. Chem. Phys. 105, 9258 (1996).

[52] R. Redmer and G. Röpke, Physica 130A, 523 (1985).

[53] A. Y. Potekhin, Phys. Plasmas 3, 4156 (1996).

[54] W. R. Hindmarsh, A. D. Petford, G. Smith, Proc. Roy. Soc. London A297, 296 (1967).

[55] G. Magni and I. Mazzitelli, Astron. Astrophys. 72, 134 (1979); D. Mihalas, W. Däppen, and D. G. Hummer, ApJ 331, 815 (1988); D. Saumon and G. Chabrier, Phys. Rev. A 44, 5122 (1991); H. Reinholz, R. Redmer, and S. Nagel, Phys. Rev E 52, 5368 (1995); F. J. Rogers, F. J. Swenson, and C. A. Iglesias, ApJ 456, 902 (1996). 